\title{
A Case Report on Neonatal Hepatoblastoma
}

\author{
Thomas ${ }^{*}$, George CR and Thomas P \\ Department of Pediatrics and Department of Pharm. D, Lisie Hospital, Kerala, India
}

*Corresponding author: Thomas J, Consultant Paediatrician, Lisie Hospital, Ernakulam, Kerala, India, 682018, Tel: +919739759723, E-mail: chinnureeba93@gmail.com

Citation: Thomas J, George CR, Thomas P (2018) A Case Report on Neonatal Hepatoblastoma. J Pediatr Dis Neonatal Care 1: 103

Article history: Received: 03 January 2018, Accepted: 05 March 2018, Published: 06 March 2018

\begin{abstract}
Neonatal hepatoblastoma is a rare primary malignant tumor. $90 \%$ of hepatoblastomas in pediatric population is seen below the age of four. Plasma alpha protein dosage is a sensitive cum specific marker for confirming the diagnosis. Low birth weight premature babies besides genetic predispositions are at higher risk to suffer from hepatoblastoma. Thrombocytosis, Beckwith-Weidemann syndrome and hemihypertrophy are also common associations. Etiology of hepatoblastoma is not known completely. We describe a case report on prenatally identified hepatoblastoma in a term male baby who underwent emergency laporotomy for resection.
\end{abstract}

Keywords: Hepatoblastoma; Alpha Feta Protein (AFP); Beckwith-Weidemann syndrome

\section{Introduction}

Hepatoblastoma constitutes $79 \%$ of all liver tumors in children with an annual incidence of 1.2 cases per million in children [1]. $90 \%$ of hepatoblastomas in pediatric population is seen below the age of four [2]. Plasma alpha protein dosage is a sensitive cum specific marker for the early diagnosis and monitoring of hepatoblastoma. There are reported cases of prenatally identified fetal hepatoblastoma [3-6]. The salient feature in all these cases is the sonographic finding of large fetal abdominal mass. Extensive hemmorhage within the tumor was a cause for fetal death in these cases. Focal nodular hyperplasia, adenoma, hepatic cysts, metastatic tumors and a fetus in fetu are the hepatic tumors other than hepatoblastoma [4]. Even though it is very rare, metastasis of fetal neuroblastoma to the liver in utero and maternal malignancy metastatic to the fetal liver are also reported $[4,7,8]$. Anemia, moderate leukocytosis and thrombocytosis along with upper quadrant abdominal mass and abdominal distension are the common symptoms of hepatic neoplasms.

Hepatoblastoma is not so prevalent in south India compared to the western countries. The most unique feature of this case is treatment of hepatoblastoma was only by resection neither by any chemotherapy treatment. Though the sensitivity and specificity of resection therapy is limited in the early diagnosis of congenital hepatoblastoma this is highly significant in our case. Surgical removal of the tumor, neoadjuvant chemotherapy especially platinum based therapy prior to tumor removal, and liver transplantation is the best treatment for neonatal hepatoblastoma.

The presence of metastases is the strongest predictor of a poor prognosis. Low birth weight premature babies besides genetic predispositions are at higher risk to suffer from hepatoblastoma [9]. Here we illustrate a case of prenatally identified hepatoblastoma in a term male baby.

\section{Case Report}

A male neonate was born at 36 weeks of gestational age by caesarean section with a birth weight of $3.050 \mathrm{~kg}$. Apgar score was $9 / 10$ at one minute and $10 / 10$ at 5 minutes. He was shifted to Neonatal ICU in view of antinatally detected abdominal mass by ultrasound. Baby developed respiratory distress at 4 hour age associated with tachypnea, and abdominal distension. Baby was intubated immediately as respiratory distress worsened. Postnatal scan was done soon after stabilizing the baby and it showed thick walled echogenic hepatic mass with irregular areas of cystic degeneration. Baby was taken up for emergency laparotomy on the same day itself. Operative finding was large friable mass measuring $6.3 \star 5.8^{\star} 5.3 \mathrm{cms}$ arising from right lobe of liver, capsule of the mass intact with altered blood clots around the mass. Histopathology report was suggestive of hepatoblastoma of fetal pattern. Baby was treated with intravenous fluids, IV antibiotics and other supportive care. Also he received blood transfusions for low 
haemoglobin. Septic work up and blood cultures were negative. IV antibiotics continued for 7 days. Alpha feta protein was 520 ng/ $\mathrm{ml}$ on day one. On day 5 ALP increased to $42000 \mathrm{ng} / \mathrm{ml}$ and decreased later on. MRI chest and abdomen done on day $12 \mathrm{showed}$ no evidence of metastasis. No association with Beckwith-Weidmann syndrome, hypoglycaemia and other congenital anomalies were seen. Oncology consultation opined regular follow up and close monitoring of ALP. Baby was discharged on day 13 on stable condition. Periodic checkups such as ultrasound scanning and serum ALP were carried out under pediatrician until the baby reached 3.5 years with no evidence of residual lesion (Figure 1). The baby has been on regular checkups since then and doing well.

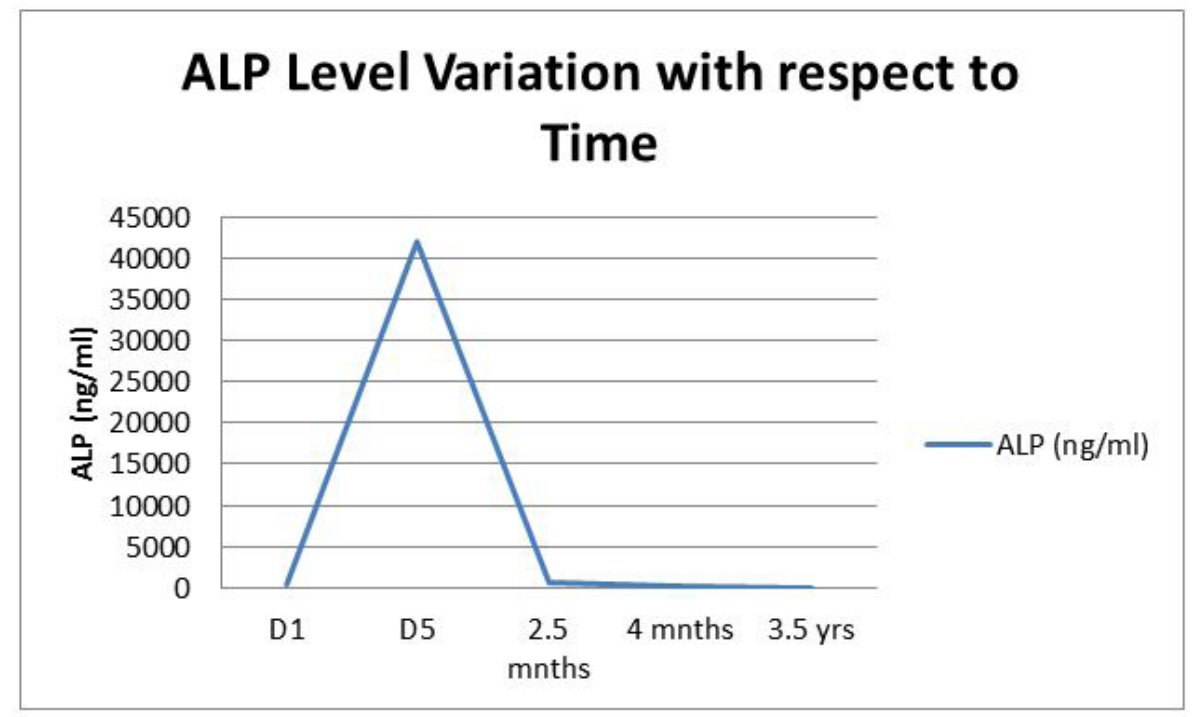

Figure 1: ALP Level Variation with respect to Time

\section{Discussion}

Fetal hepatoblastoma has higher rates of survival and low rates of morbidity today. Complete resection of the tumor and adjuvant chemotherapy give 100\% survival rate generally. Favourable histology and low mitotic rate with complete resection may avoid the necessity of chemotherapy [1]. Here the neonate had not undergone pre or post chemotherapy rather complete resection was achieved post surgically. The most unique feature of this case is treatment of hepatoblastoma is only by resection neither by any chemotherapy treatment. A Japanese literature describes a case of uneventful recovery of neonatal hepatoblastoma without postoperative adjuvant chemotherapy [10].

Platinum based therapy, using cisplatin or carboplatin along with vincristine or 5-flurouracil is the mainstay of chemotherapy treatment for hepatoblastoma. Preoperative chemotherapy completely eradicates metastatic pulmonary disease and multinodular liver disease. Radiotherapy is preferred if resection margins show microscopic disease. The commonly seen adverse reactions of cisplatin are ototoxicity. Murawski, et al., presented the findings of two prospective studies of hepatoblastoma conducted and these trials evaluated whether modified platinum- and doxorubicin-based chemotherapy increases tumor resectability and changes patient outcomes and it is concluded that intensified use of platinum agents did not improve the survival of patients with hepatoblastoma and that new approaches to treatment are required for the coming future [11].

Complete resection of hepatoblastoma through surgery can be achieved in approximately one third of patients, in case of stage I or II disease. But this is influenced by age of the patient at diagnosis, size of the tumor and practical knowledge of the surgical staff. Initial resection of operable primary tumors by lobectomy is the standard of care. Nodal involvement is rare. Intraoperative hemorrhage is the most often complication of surgery. Liver transplantation is considered when the disease is unresectable even after neoadjuvant or adjuvant chemotherapy [12].

A study indicates intrauterine and or neonatal factors like low birth weight and asphyxia are associated with increased risk of hepatoblastoma. They also quote the probability of these factors as a consequence of hepatoblastoma developing in utero [13]. In contrast there are indications of an increased risk of hepatoblastoma in children with high birth weight compared to normal birth weight $[14,15]$. Plausible explanations could be greater number of cells resulting in more cell divisions or proliferation of cancer cells in utero due to insulin-like growth factor 1 . This baby had a birth weight of $3.050 \mathrm{~kg}$ with an apgar score of 9/10 at one minute.

There are some genetic conditions to be associated with risk of developing hepatoblastoma such as Beckwith-Wiedemann syndrome, hemi hypertrophy, APC-associated polyposis, a-1-antitrypsin deficiency and some metabolic disorders including tyrosinemia, galactosemia and glycogen storage disease type 1 [16].

Etiology of hepatoblastoma is not fully known. There are evidences suggesting hepatoblastoma derived from pluripotent stem cell [17]. This could be due to developmental error during hepatogenesis. Hepatobalstoma affect right lobe of liver more often than left lobe. Loss of heterozygosity (LOH) of chromosome arm 11p markers, familial adenomatous polyposis (FAP), loss of function mutations in APC, abnormal regulations in Wnt signalling pathway are some of the suggested mechanisms. Thrombocytosis, prematurity, Beckwith-Weidemann syndrome, hemihypertrophy amd low birth weight are common associations. Most of the 
reported cases of hepatoblastoma possess an association with Beckwith-Weidemann syndrome which was absent in our case. It is advised to make tumor surveillance mandatory during neonatal period if syndrome overgrowth patterns are recognized. If hepatoblastoma is diagnosed antenatal it is recommended to undergo caesarean section instead of vaginal delivery as normal vaginal may cause tumor rupture.

There are mainly two types of hepatoblastoma epithelial and mesenchymal -epithelial type having four different subtypes - fetal, embryonic, small cell type and macrotrabecular. Hepatoblastoma can be diagnosed on cytology and these subtypes are important for the prognostic features [18].

The most useful laboratory test for hepatoblastoma is serum AFP. AFP is built from the fetal liver and yolk sac. The levels of AFP decline to adult values during the first 6 months after birth. It is less than $10 \mathrm{ng} / \mathrm{ml}$ in normal adults. The tumor marker for screening and confirmation of diagnosis is AFP, but it is not significant or specific in all the cases. Serum AFP levels can also be increased in hepatocellular carcinoma, hepatitis, cirrhosis, germ cell tumor, hemangioendothelioma, testicular tumor and gallbladder carcinoma. In Small cell type hepatoblastoma, serum AFP may not rise because of less differentiation of the cells, but in advanced differentiated types like fetal HBL, serum AFP is usually elevated. But in this particular case was of fetal type, AFP was marginally elevated [19].

In our study, AFP value was found to be elevated and was highly advantageous and significant as it could be used for follow-up. But serum AFP level may not be elevated in some cases of hepatoblastoma and histology is necessary in such cases [20].

Serum alpha protein (AFP) is a remarkable factor in identifying hepatoblastoma and hepatocellular carcinoma. High AFP levels are seen in $90 \%$ cases of hepatoblastoma [21]. AFP level in this case was $42000 \mathrm{ng} / \mathrm{ml}$ in the first few days and decreased to 142 $\mathrm{ng} / \mathrm{ml}$ after four months. Tumor involvement of liver is a possibility for recurrent hypogycemia during neonatal period even after initial stabilization of blood sugar values. Hypoglycemia was the presenting symptom of hepatoblastoma in one of the cases described in the report by Smith, et al. [22].

Aggressive tumor like fetal hepatoblastoma requires chemotherapy to shrink the tumor size and then surgery. But in our case complete resection was gained through surgical excision in spite of perplexity. Patients die even after chemotherapy due to postoperative complications, CMV hepatitis, myelodysplastic syndrome and rectal cancer. The rupture of tumor can lead to metastasis of other organs.

\section{Conclusion}

Diagnosis and treatment of neonatal liver tumors are challenging. Hepatoblastoma, due to inconclusive radiological findings can be misinterpreted as infantile hemangioendothelioma. Serum AFP is an eminent factor in identifying hepatoblastoma. Detailed checkups during pregnancy may help in early diagnosis. Favorable survival outcome could be achieved with complete tumor excision and adjuvant chemotherapy.

\section{References}

1. Darbari A, Sabin KM, Shapiro CN, Schwarz KB (2003) Epidemiology of primary hepatic malignancies in U.S. children. Hepatology 38: 560-6.

2. Mussa A, Ferrero GB, Ceoloni B, Basso E, Chiesa N, et al. (2011) Neonatal hepatoblastoma in a newborn with severe phenotype of Beckwith-Wiedemann syndrome. Eur J Pediatr 170: 1407-11.

3. Kazzi NJ, Chang CH, Roberts EC, Shankaran S (1989) Fetal hepatoblastoma presenting as nonimmune hydrops. Am J Perinatol 6: 278-80.

4. Catanzarite V, Hilfiker M, Daneshmand S, Willert J (2008) Prenatal Diagnosis of Fetal Hepatoblastoma. Val Catanzarite Case Report and Review of the Literature. J Ultrasound Med 27:1095-8

5. Shih JC, Tsao PN, Huang SF, Yen BLJ, Lin JH, et al. (2009) Antenatal diagnosis of congenital hepatoblastoma in utero. Ultrasound Obstet Gynecol 16: 94-7.

6. Aviram R, Cohen IJ, Kornreich L, Braslavski D, Meizner I (2005) Prenatal imaging of fetal hepatoblastoma. J Matern Fetal Neonatal Med 17: 157-9.

7. Woodward PJ, Sohaey R, Kennedy A, Koeller KK (2005) A comprehensive review of fetal tumors with pathologic correlation. Radiographics 25: 215-42.

8. Alexander A, Samlowski WE, Grossman D, Bruggers CS, Harris RM, et al. (2003) Metastatic melanoma in pregnancy: risk of transplacental metastases in the infant. J Clin Oncol 21: 2179-86.

9. Comert S, Vitrinel A, Akin Y, Mutlu GY, Tokuc G et al. (2007) Abdominal mass in a neonate: Hepatoblastoma. Clinical brief. Indian Journal of Paediatrics 74: 956-8.

10. Honzumi M, Miura T, Fujino I, Suzuki H (1990) Hepatoblastoma in neonates: Report of a case and review of the Japanese literature. Jpn J Surg 20: 331-4.

11. Murawski M, Weeda VB, Maibach R, Morland B, Roebuck DJ, et al. (2016) Hepatocellular carcinoma in children: Does modified platinum- and doxorubicinbased chemotherapy increase tumor resectability and change outcome? Lessons learned from the SIOPEL 2 and 3 studies. J Clin Oncol 34:1050-6.

12. Jennifer RW, Gary D, Alexander A, Arun A R (2017) Pediatric Hepatoblastoma treatment and management. Drugs \& Diseases, Pediatrics: general medicine. Medscape.

13. de Fine Licht S, Schmidt LS, Rod NH, Schmiegelow K, Lähteenmäki PM, et al. (2012) Hepatoblastoma in the Nordic countries. Int. J Cancer 131: E555-61.

14. Reynolds P, Urayama KY, von Behren J, Feusner J (2004) Birth characteristics and hepatoblastoma risk in young children. Cancer 100: 1070-6.

15. McLaughlin CC, Baptiste MS, Schymura MJ, Nasca PC, Zdeb MS (2006) Maternal and infant birth characteristics and hepatoblastoma. Am J Epidemiol 163: 818-28.

16. Puchmajerová A, Křepelová A, Indráková J, Sítková R, Balaščak I, et al. (2016) Hepatoblastoma, Etiology, Case Reports. Klin Onkol 29: S78-82. 
17. Ruck P, Xiao JC (2002) Stem-like cells in hepatoblastoma. Med Pediatr Oncol 39: 504-7.

18. Iyer VK, Kapila K, Agarwala S, Verma K (2005) Fine needle aspiration cytology of hepatoblastoma. Recognition of subtypes on cytomorphology. Acta Cytol 49: 355-64.

19. Tsuchida Y, Ikeda H, Suzuki N, Takahashi A, Kuroiwa M, et al. (1999) A case of well-differentiated, fetal-type hepatoblastoma with very low serum alphafetoprotein. J Pediatr Surg 34: 1762-4.

20. Sarbani C, Sumana M, Anup B, Abhishek S, Biswas SK (2012) Hepatoblastoma in the neonatal period: An unusual presentation. J Cytol 29: 252-4.

21. Christine UCL, James G (2014) Mayo Clinic Body MRI Case Review. Oxford University, USA.

22. Smith AC, Shuman C, Chitayat D, Steele L, Ray PN, et al. (2007) Severe presentation of Beckwith-Wiedemann syndrome associated with high levels of constitutional paternal uniparental disomy for chromosome 11p15. Am J Med Genet A 143A: 3010-5. 